\title{
Accuracy of methods for diagnosing heart diseases in cats
}

\author{
Tanarut Laudhittirut, Natrada Rujivipat, Kornnicha Saringkarisate, Peeraya Soponpattana, Teerawat Tunwichai and \\ Sirilak Disatian Surachetpong iD
}

\author{
Department of Veterinary Medicine, Faculty of Veterinary Science, Chulalongkorn University, Bangkok 10330, Thailand. \\ Corresponding author: Sirilak Disatian Surachetpong, e-mail: sirilakd27@gmail.com \\ Co-authors: TL: tanarut.lau@gmail.com, NR: natrada.ruj@hotmail.com, KS: kornnicha.s@hotmail.com, \\ PS: pearpeeraya@gmail.com, TT: t.tunwichai@gmail.com
}

Received: 14-11-2019, Accepted: 07-04-2020, Published online: 09-05-2020

doi: www.doi.org/10.14202/vetworld.2020.872-878 How to cite this article: Laudhittirut T, Rujivipat N, Saringkarisate K, Soponpattana P, Tunwichai T, Surachetpong SD (2020) Accuracy of methods for diagnosing heart diseases in cats, Veterinary World, 13(5): 872-878.

\begin{abstract}
Aim: This study aimed to determine the accuracy of the current methods for diagnosing heart diseases in cats.

Materials and Methods: The data of 58 cats were retrospectively retrieved. Cats were classified into two groups: Thirtyeight cats with heart diseases and 20 healthy cats. Echocardiography was the gold standard method for diagnosing heart disease. The results of seven methods were retrieved: (1) Vertebral heart score (VHS) with a cutoff value $>8$, (2) VHS with a cutoff value $>8.5$, (3) multiplication of cardiac length (L) and width (W), (4) multiplication of cardiac L and W divided by the L of the fourth sternal thoracic bone, (5) N-terminal Pro-B-type natriuretic peptide (NT-proBNP) point-of-care test, (6) subjective ultrasonographic assessment of the left atrial size, and (7) subjective radiographic assessment of the left atrial size. Cross-tabulation was used to calculate the sensitivity, specificity, accuracy, positive predictive value, and negative predictive value for each test. This study found that using the NT-proBNP point-of-care test was optimal in the diagnosis of cats with heart disease.
\end{abstract}

Results: The subjective ultrasonographic assessment of the left atrial size was good for diagnosing hypertrophic cardiomyopathy and congestive heart failure.

Conclusion: This study showed that the more tests used, the higher the reliability of the diagnosis.

Keywords: cardiac disease diagnosis, N-terminal Pro-B-type natriuretic peptide, thoracic radiography, ultrasonography.

\section{Introduction}

Heart disease is common in cats, affecting $10 \%-15 \%$ of all cats $[1,2]$. Most cats affected by heart disease may not show clinical signs. A few cats may develop signs of congestive heart failure $(\mathrm{CHF})$ or suddenly die [3]. Common heart diseases in cats include cardiomyopathies, such as hypertrophic cardiomyopathy (HCM), restrictive cardiomyopathy (RCM), and unclassified cardiomyopathy (UCM). Congenital cardiac disorders in cats, such as atrioventricular valve dysplasia and septal defects, are less common [4].

Echocardiography is the gold standard method for diagnosing heart disease in cats. Despite being a definitive method, performing echocardiography requires experienced sonographers. Furthermore, the required equipment is expensive. Therefore, echocardiography is difficult to access and unavailable in primary care animal hospitals in some regions. At present, there are three common methods for diagnosing heart diseases in cats: Radiography, thoracic focused assessment with sonography for trauma (TFAST), and cardiac

Copyright: Laudhittirut, et al. Open Access. This article is distributed under the terms of the Creative Commons Attribution 4.0 International License (http://creativecommons.org/licenses/ by/4.0/), which permits unrestricted use, distribution, and reproduction in any medium, provided you give appropriate credit to the original author(s) and the source, provide a link to the Creative Commons license, and indicate if changes were made. The Creative Commons Public Domain Dedication waiver (http:// creativecommons.org/publicdomain/zero/1.0/) applies to the data made available in this article, unless otherwise stated. biomarker measurements. These methods may help in diagnosing heart disease in cats, particularly in some locations where comprehensive echocardiography is unavailable. Radiography is the most common method used to evaluate cardiac size and shape. However, there is a limitation in using radiography in diagnosing heart disease in cats. Some types of heart disease in cats, such as cardiomyopathies, may not cause cardiac enlargement that can be detected on radiography. The VHS, a method in evaluating the size of the heart on radiography, may not distinguish cats with and without heart disease. The cutoff value of VHS for cats with heart disease is eight. Cats with a VHS $>9.3$ have an extremely high tendency of having heart disease. However, cats with VHS 8-9.3 are classified into a grey zone and need further investigation for the diagnosis of heart disease [5]. A new method for detecting heart diseases in cats using thoracic radiography has been published [6]. This method has $82.2 \%$ sensitivity and $66.9 \%$ specificity in differentiating cardiac and noncardiac diseases in cats. TFAST can also be used. This technique is a reliable method in assessing cardiac structural changes without the technical challenge and long time. Left atrial enlargement can be detected using TFAST. A left atrial enlargement is highly suggestive of a restrictive filling pattern, which is an indicator in the diagnosis of heart diseases in cats [7]. The measurement of cardiac biomarkers is another method in diagnosing heart disease in cats. 
Pro-B-type natriuretic peptide (proBNP) is a substance that is released from cardiac muscles when the heart is stretched or stressed [8]. At present, an N-terminal proBNP (NT-proBNP) point-of-care test kit is available. However, the test is semiquantitative and has some limitations. Noncardiac diseases, such as severe respiratory or kidney diseases (with creatinine level $>2.8 \mathrm{mg} / \mathrm{d}$ ), increase the likelihood of false-positive results [9]. An increase in NT-proBNP levels can be observed in cats with severe arrhythmias, pulmonary hypertension, systemic hypertension, and hyperthyroidism $[10,11]$.

This study aimed to determine the accuracy of current methods for diagnosing heart diseases, HCM, and $\mathrm{CHF}$ in cats.

\section{Materials and Methods}

\section{Ethical approval}

Ethical approval is not necessary because of the retrospective design of the study.

\section{The data of cats}

The medical records of cats presented to our university, from 2016 to 2018 were retrospectively reviewed. The data of cats that had undergone complete cardiac examinations, including clinical evaluations, cardiac biomarker measurements, radiography, and echocardiography, were collected. The data from cats with concurrent diseases affecting NT-proBNP levels, including hyperthyroidism, systemic hypertension, kidney diseases (creatinine level $>2.8 \mathrm{mg} / \mathrm{dL}$ ), and respiratory diseases, were excluded from the study. Echocardiography was used as the gold standard method for diagnosing cardiac diseases.

Digital thoracic radiographs were archived. Radiographs with pleural effusion, pneumothorax, and unclear cardiac silhouette were excluded from the study. The length (L) of the long axis (distance from the ventral border of the carina to the cardiac apex) and width $(\mathrm{W})$ (maximum cardiac width perpendicular to the measurement of the long axis) of the heart were measured on the right lateral view. Five individuals performed each measurement. The interobserver coefficient of variation was calculated for each measurement method. The VHS was evaluated. Two measurement methods from a previous study [6] were performed:

1. Multiplication of $\mathrm{L}$ and $\mathrm{W}(\mathrm{L} \times \mathrm{W})$ with the cutoff value $>16.46 \mathrm{~cm}^{2}$.

2. Multiplication of $\mathrm{L}$ and $\mathrm{W}$ divided by the length of the fourth sternal thoracic bone $(\mathrm{S} 4)(\mathrm{L} \times \mathrm{W} / \mathrm{S} 4)$ with a cutoff value $>12.85 \mathrm{~cm}$.

The result of a feline NT-proBNP point-of-care test kit $\left(\mathrm{SNAP}^{\circledR}\right.$ Feline proBNP, IDEXX, Maine, United States) was recorded as positive or negative. All results were interpreted using the automated test kit reader machine (SNAP ${ }^{\circledR}$ Pro Analyzer, IDEXX, Maine, United States).

The result of the left atrial enlargement subjectively assessed by TFAST was recorded as normal or enlarged size.
Echocardiography was performed using an ultrasound machine (EKO7, Samsung Medison, Seoul, South Korea) with 2-4 and 4-12 multifrequency $\mathrm{MHz}$ phased array transducers. The definitive heart disease diagnosed by echocardiography was recorded. The left atrial size measured from the right parasternal cross-sectional view at the level of the left atrium was noted. A left atrial size $>16 \mathrm{~mm}$ was classified as enlargement [7]. The diagnostic criteria for HCM included increased wall thickness ( $\geq 6 \mathrm{~mm}$ ) and decreased left ventricular chamber size [11]. RCM was identified by left atrial or biatrial enlargement, normal left ventricular wall thickness, normal or decreased systolic function, and restrictive ventricular filling pattern with pulsed-wave Doppler echocardiography [12]. Congenital heart disease was classified according to a previously published study [13]. Ventricular septal defects were diagnosed using two-dimensional (2D) and color Doppler echocardiography to identify flow across the lesion. Mitral and tricuspid valve dysplasias were diagnosed based on the presence of abnormalities of the valve apparatus and valvular regurgitation on 2D and color Doppler echocardiography.

\section{Statistical analysis}

Statistical analysis was performed using a commercial statistical program (SPSS software Version 22, IBM Inc., NY, USA) and cross-tabulation to determine the specificity, sensitivity, and accuracy of each test. The positive predictive value (PPV) and negative predictive value (NPV) were also calculated.

\section{Results}

The data and radiographs of 58 cats were retrieved. Twenty-nine cats were male. Breeds of the cats included Domestic Shorthair $(n=32)$, Persian $(n=15)$, African Shorthair $(n=4)$, Scottish Fold $(n=2)$, Sphinx $(n=1)$, Siamese $(n=1)$, Khao Manee $(n=1)$, Maine Coon $(n=1)$, and British Shorthair $(n=1)$. Twenty cats (ten males and ten females) were normal. Thirty-eight cats (twenty males and 18 females) had heart diseases, including HCM $(n=24), \operatorname{RCM}(n=5)$, UCM $(n=3)$, hypertrophic obstructive cardiomyopathy $(n=3)$, atrial septal defect $(n=1)$, mitral valve dysplasia $(n=1)$, and tricuspid valve dysplasia $(n=1)$.

Seven diagnostic methods were performed in all cats: VHS at cutoff values $>8$ and $>8.5$, multiplication of the length and width of the heart $(\mathrm{L} \times \mathrm{W})$, ratio of the multiplication of the length and width of the heart to the length of the fourth sternal thoracic bone $(\mathrm{L} \times \mathrm{W} / \mathrm{S} 4)$, subjective radiographic assessment of left atrial size (AX), NT-proBNP point-of-care test (NT-proBNP), and subjective ultrasonographic assessment of the left atrial size (AU). Interobserver variability of $\mathrm{VHS}, \mathrm{L} \times \mathrm{W}$, and $\mathrm{L} \times \mathrm{W} / \mathrm{S} 4$ were $6.63 \%$, $6.95 \%$, and $5.66 \%$, respectively.

The specificity, sensitivity, accuracy, PPV, and NPV of each diagnostic test and combination 
tests were analyzed using the diagnostic result from echocardiography as the gold standard method. The accuracy of the tests to distinguish cats with and without heart diseases, cats with and without HCM, and cats with and without CHF is summarized in Tables-1-3, respectively.

The best method for diagnosing cats with any heart disease was the NT-proBNP point-of-care test.

Table-1: Specificity, sensitivity, accuracy, positive predictive, and negative predictive values for each test in cats with $(n=38)$ and without heart disease $(n=20)$.

\begin{tabular}{|c|c|c|c|c|c|c|}
\hline Parameters & $\begin{array}{c}\text { Number positive/ } \\
\text { negative }\end{array}$ & $\begin{array}{c}\text { Sensitivity } \\
(\%)\end{array}$ & $\begin{array}{c}\text { Specificity } \\
(\%)\end{array}$ & $\begin{array}{c}\text { Accuracy } \\
(\%)\end{array}$ & PPV & NPV \\
\hline \multicolumn{7}{|l|}{ One test } \\
\hline VHS $>8$ & $24 / 34$ & 44.74 & 65.00 & 51.72 & 70.83 & 38.24 \\
\hline VHS $>8.5$ & $13 / 45$ & 26.32 & 85.00 & 46.55 & 76.92 & 37.78 \\
\hline $\mathrm{L} \times \mathrm{W}$ & $24 / 34$ & 63.16 & 50.00 & 58.62 & 70.59 & 41.67 \\
\hline $\mathrm{L} \times \mathrm{W} / \mathrm{S} 4$ & $25 / 33$ & 42.10 & 55 & 46.55 & 64 & 33.33 \\
\hline$A X$ & $29 / 29$ & 60.53 & 70.00 & 44.65 & 79.31 & 48.28 \\
\hline NT-proBNP & $30 / 28$ & 68.42 & 80.00 & 72.41 & 86.67 & 57.14 \\
\hline$A U$ & $16 / 42$ & 42.10 & 100 & 62.07 & 100 & 47.62 \\
\hline \multicolumn{7}{|l|}{ Two tests } \\
\hline $\mathrm{VHS}>8+\mathrm{L} \times \mathrm{W}$ & $18 / 18$ & 56.52 & 61.54 & 58.33 & 72.22 & 44.44 \\
\hline $\mathrm{VHS}>8+\mathrm{L} \times \mathrm{W} / \mathrm{S} 4$ & $15 / 24$ & 40.74 & 66.67 & 48.72 & 73.33 & 33.33 \\
\hline $\mathrm{VHS}>8+\mathrm{AX}$ & 24/19 & 53.57 & 73.33 & 60.47 & 78.95 & 45.83 \\
\hline VHS $>8+N T$-proBNP & $15 / 19$ & 61.9 & 84.62 & 70.59 & 86.67 & 57.89 \\
\hline $\mathrm{VHS}>8+\mathrm{AU}$ & $11 / 29$ & 40.74 & 100 & 60 & 100 & 44.83 \\
\hline $\mathrm{VHS}>8.5+\mathrm{L} \times \mathrm{W}$ & $11 / 22$ & 40 & 76.92 & 54.55 & 72.73 & 45.45 \\
\hline VHS $>8.5+L \times W / S 4$ & $9 / 28$ & 25 & 78.57 & 44.74 & 66.67 & 37.93 \\
\hline VHS $>8.5+A X$ & $13 / 29$ & 40 & 82.35 & 57.14 & 76.92 & 48.28 \\
\hline VHS $>8.5+N T-p r o B N P$ & $9 / 24$ & 44.44 & 93.33 & 66.67 & 88.89 & 58.33 \\
\hline $\mathrm{VHS}>8.5+\mathrm{AU}$ & $7 / 36$ & 26.92 & 100 & 55.81 & 100 & 47.22 \\
\hline $\mathrm{L} \times \mathrm{W}+\mathrm{AX}$ & $21 / 15$ & 69.57 & 64.29 & 67.57 & 76.19 & 56.25 \\
\hline $\mathrm{L} \times \mathrm{W}+\mathrm{NT}-$ proBNP & $18 / 12$ & 77.27 & 87.5 & 80 & 94.44 & 58.33 \\
\hline$L \times W+A U$ & $15 / 23$ & 53.57 & 100 & 65.79 & 100 & 43.48 \\
\hline $\mathrm{L} \times \mathrm{W} / \mathrm{S} 4+\mathrm{AX}$ & $17 / 21$ & 52 & 69.23 & 57.89 & 76.47 & 42.85 \\
\hline L×W/S4+NT-proBNP & $14 / 17$ & 60 & 81.82 & 67.74 & 85.71 & 52.94 \\
\hline $\mathrm{L} \times \mathrm{W} / \mathrm{S} 4+\mathrm{AU}$ & $12 / 29$ & 40 & 100 & 56.1 & 100 & 37.93 \\
\hline$A X+N T-p r o B N P$ & $19 / 19$ & 70.83 & 85.71 & 76.32 & 89.47 & 63.16 \\
\hline$A X+A U$ & $12 / 25$ & 52.17 & 100 & 70.27 & 100 & 56 \\
\hline AU+NT-proBNP & $13 / 25$ & 59.09 & 100 & 76.32 & 100 & 64 \\
\hline \multicolumn{7}{|l|}{ Three tests } \\
\hline VHS $>8+L \times W+A X$ & $15 / 13$ & 66.67 & 63.64 & 65.52 & 75 & 53.85 \\
\hline VHS $>8+L \times W+N T-p r o B N P$ & $12 / 9$ & 78.57 & 85.71 & 80.95 & 91.67 & 66.67 \\
\hline $\mathrm{VHS}>8+\mathrm{L} \times \mathrm{W}+\mathrm{AU}$ & $11 / 17$ & 55 & 100 & 67.86 & 100 & 47.06 \\
\hline $\mathrm{VHS}>8+\mathrm{L} \times \mathrm{W} / \mathrm{S} 4+\mathrm{AX}$ & $13 / 18$ & 47.62 & 70 & 54.84 & 76.92 & 38.89 \\
\hline VHS $>8+L \times W / S 4+N T-p r o B N P$ & $11 / 12$ & 66.67 & 87.5 & 73.91 & 90.91 & 58.33 \\
\hline $\mathrm{VHS}>8+\mathrm{L} \times \mathrm{W} / \mathrm{S} 4+\mathrm{AU}$ & $10 / 22$ & 43.48 & 100 & 58.06 & 100 & 38.1 \\
\hline VHS $>8+A X+N T-p r o B N P$ & $13 / 16$ & 66.67 & 90.91 & 75.86 & 92.31 & 62.5 \\
\hline $\mathrm{VHS}>8+\mathrm{AX}+\mathrm{AU}$ & $10 / 21$ & 50 & 100 & 67.74 & 100 & 52.38 \\
\hline VHS $>8+A U+N T-p r o B N P$ & $10 / 17$ & 62.5 & 100 & 77.78 & 100 & 64.71 \\
\hline VHS $>8.5+L \times W+A X$ & $9 / 16$ & 53.33 & 75 & 62.96 & 72.73 & 56.25 \\
\hline VHS $>8.5+L \times W+N T-p r o B N P$ & $8 / 11$ & 63.64 & 87.5 & 73.68 & 87.5 & 63.64 \\
\hline VHS $>8.5+L \times W+A U$ & $7 / 21$ & 38.89 & 100 & 60.71 & 100 & 47.62 \\
\hline $\mathrm{VHS}>8.5+\mathrm{L} \times \mathrm{W} / \mathrm{S} 4+\mathrm{AX}$ & $8 / 21$ & 33.33 & 75 & 50 & 66.67 & 42.86 \\
\hline VHS $>8.5+L \times W / S 4+N T-p r o B N P$ & $7 / 14$ & 50 & 90 & 68.18 & 85.71 & 60 \\
\hline $\mathrm{VHS}>8.5+\mathrm{L} \times \mathrm{W} / \mathrm{S} 4+\mathrm{AU}$ & $6 / 26$ & 28.57 & 100 & 53.13 & 100 & 42.31 \\
\hline VHS $>8.5+A X+N T-p r o B N P$ & $9 / 19$ & 53.33 & 92.31 & 71.43 & 88.89 & 63.16 \\
\hline $\mathrm{VHS}>8.5+\mathrm{AX}+\mathrm{AU}$ & $7 / 25$ & 38.89 & 100 & 65.63 & 100 & 56 \\
\hline VHS $>8.5+A U+N T-p r o B N P$ & $7 / 21$ & 50 & 100 & 75 & 100 & 66.67 \\
\hline $\mathrm{L} \times \mathrm{W}+\mathrm{AX}+\mathrm{NT}-$ proBNP & $14 / 10$ & 81.25 & 87.5 & 83.33 & 92.86 & 70 \\
\hline$L \times W+A X+A U$ & $12 / 15$ & 66.67 & 100 & 77.78 & 100 & 60 \\
\hline $\mathrm{L} \times \mathrm{W}+\mathrm{AU}+\mathrm{NT}-\mathrm{proBNP}$ & $12 / 12$ & 76.47 & 100 & 83.33 & 100 & 63.64 \\
\hline $\mathrm{L} \times \mathrm{W} / \mathrm{S} 4+\mathrm{AX}+\mathrm{NT}-$ proBNP & $13 / 12$ & 68.75 & 77.78 & 72 & 84.62 & 58.33 \\
\hline $\mathrm{L} \times \mathrm{W} / \mathrm{S} 4+\mathrm{AX}+\mathrm{AU}$ & $11 / 18$ & 55 & 100 & 68.97 & 100 & 50 \\
\hline $\mathrm{L} \times \mathrm{W} / \mathrm{S} 4+\mathrm{AU}+\mathrm{NT}-\mathrm{proBNP}$ & $11 / 16$ & 58.82 & 100 & 73.08 & 100 & 56.25 \\
\hline$A X+A U+N T-p r o B N P$ & $10 / 17$ & 62.5 & 100 & 78.57 & 100 & 66.67 \\
\hline
\end{tabular}

$\mathrm{AX}=$ The subjective radiographic assessment of the left atrial size, $\mathrm{AU}=$ The ultrasonographic measurement of the left atrial diameter, $L \times W=$ The multiplication of the $L$ and the $W$ of the heart, $L \times W / S 4=$ The ratio of the multiplication of the $L$ and the $W$ of the heart to the $L$ of the fourth sternal thoracic bone, NPV $=$ Negative predictive value, NT-proBNP $=$ The NT-proBNP point-of-care test, PPV $=$ Positive predictive value, VHS $>8=$ Vertebral heart score at a cutoff value of $>8$, VHS $>8.5=$ Vertebral heart score at a cutoff value of $>8.5$ 
Table-2: Specificity, sensitivity, accuracy, positive predictive, and negative predictive values of tests for distinguishing cats with $(n=16)$ and without hypertrophic cardiomyopathy $(n=42)$.

\begin{tabular}{|c|c|c|c|c|c|c|}
\hline Parameters & $\begin{array}{c}\text { Number positive/ } \\
\text { negative }\end{array}$ & $\begin{array}{c}\text { Sensitivity } \\
(\%)\end{array}$ & $\begin{array}{l}\text { Specificity } \\
(\%)\end{array}$ & $\begin{array}{c}\text { Accuracy } \\
(\%)\end{array}$ & PPV & NPV \\
\hline \multicolumn{7}{|l|}{ One test } \\
\hline VHS $>8$ & $24 / 34$ & 62.50 & 66.67 & 65.52 & 41.67 & 82.35 \\
\hline VHS $>8.5$ & $13 / 45$ & 43.75 & 85.71 & 74.14 & 53.85 & 80 \\
\hline $\mathrm{L} \times \mathrm{W}$ & $24 / 34$ & 62.50 & 42.86 & 48.28 & 29.41 & 75 \\
\hline $\mathrm{L} \times \mathrm{W} / \mathrm{S} 4$ & $25 / 33$ & 56.25 & 61.90 & 60.34 & 36 & 78.79 \\
\hline$A X$ & $29 / 29$ & 87.50 & 64.28 & 70.69 & 48.28 & 93.1 \\
\hline NT-proBNP & $30 / 28$ & 87.50 & 61.90 & 68.97 & 46.67 & 92.86 \\
\hline $\mathrm{AU}$ & $16 / 42$ & 62.50 & 85.71 & 79.31 & 62.5 & 85.71 \\
\hline \multicolumn{7}{|l|}{ Two tests } \\
\hline $\mathrm{VHS}>8+\mathrm{L} \times \mathrm{W}$ & $18 / 18$ & 66.67 & 58.33 & 61.11 & 44.44 & 77.78 \\
\hline $\mathrm{VHS}>8+\mathrm{L} \times \mathrm{W} / \mathrm{S} 4$ & $15 / 24$ & 63.64 & 71.43 & 69.23 & 46.67 & 83.33 \\
\hline $\mathrm{VHS}>8+\mathrm{AX}$ & $24 / 19$ & 83.33 & 70.97 & 74.42 & 52.63 & 91.67 \\
\hline VHS $>8+$ NT-proBNP & $15 / 19$ & 90 & 75 & 79.41 & 60 & 94.74 \\
\hline $\mathrm{VHS}>8+\mathrm{AU}$ & $11 / 29$ & 66.6 & 89.29 & 82.5 & 72.73 & 86.21 \\
\hline VHS $>8.5+L \times W$ & $11 / 22$ & 54.55 & 77.27 & 69.7 & 54.55 & 77.27 \\
\hline VHS $>8.5+L \times W / S 4$ & $9 / 28$ & 50 & 85.71 & 76.32 & 55.56 & 82.76 \\
\hline VHS $>8.5+A X$ & $13 / 29$ & 77.78 & 81.82 & 80.95 & 53.85 & 93.1 \\
\hline VHS $>8.5+$ NT-proBNP & $9 / 24$ & 77.78 & 91.67 & 87.88 & 77.78 & 91.67 \\
\hline $\mathrm{VHS}>8.5+\mathrm{AU}$ & $7 / 36$ & 54.55 & 96.88 & 86.05 & 85.71 & 86.11 \\
\hline$L \times W+A X$ & $21 / 15$ & 83.33 & 56 & 64.86 & 47.62 & 87.5 \\
\hline $\mathrm{L} \times \mathrm{W}+\mathrm{NT}-$ proBNP & $18 / 12$ & 100 & 54.55 & 66.67 & 44.44 & 100 \\
\hline$L \times W+A U$ & $15 / 23$ & 62.5 & 77.27 & 71.05 & 62.5 & 77.27 \\
\hline $\mathrm{L} \times \mathrm{W} / \mathrm{S} 4+\mathrm{AX}$ & $17 / 21$ & 81.82 & 70.37 & 73.68 & 52.94 & 90.48 \\
\hline L×W/S4+NT-proBNP & $14 / 17$ & 100 & 70.83 & 77.42 & 50 & 100 \\
\hline $\mathrm{L} \times \mathrm{W} / \mathrm{S} 4+\mathrm{AU}$ & $12 / 29$ & 60 & 88.46 & 78.05 & 75 & 79.31 \\
\hline$A X+N T-p r o B N P$ & $19 / 19$ & 100 & 70.37 & 78.95 & 57.89 & 100 \\
\hline$A X+A U$ & $12 / 25$ & 83.33 & 92 & 89.19 & 83.33 & 92 \\
\hline AU+NT-proBNP & $13 / 25$ & 100 & 83.33 & 86.84 & 61.54 & 100 \\
\hline \multicolumn{7}{|l|}{ Three tests } \\
\hline$V H S>8+L \times W+A X$ & $15 / 13$ & 80 & 57.89 & 65.52 & 50 & 84.62 \\
\hline VHS $>8+L \times W+N T-p r o B N P$ & $12 / 9$ & 100 & 64.29 & 76.19 & 58.33 & 100 \\
\hline $\mathrm{VHS}>8+\mathrm{L} \times \mathrm{W}+\mathrm{AU}$ & $11 / 17$ & 66.67 & 81.25 & 75 & 72.73 & 76.47 \\
\hline $\mathrm{VHS}>8+\mathrm{L} \times \mathrm{W} / \mathrm{S} 4+\mathrm{AX}$ & $13 / 18$ & 77.78 & 72.73 & 74.19 & 53.85 & 88.89 \\
\hline VHS $>8+L \times W / S 4+N T-p r o B N P$ & $11 / 12$ & 100 & 70.59 & 78.26 & 54.55 & 100 \\
\hline $\mathrm{VHS}>8+\mathrm{L} \times \mathrm{W} / \mathrm{S} 4+\mathrm{AU}$ & $10 / 22$ & 63.64 & 85 & 77.42 & 70 & 80.95 \\
\hline VHS $>8+A X+N T-p r o B N P$ & $13 / 16$ & 100 & 80 & 86.21 & 69.23 & 100 \\
\hline $\mathrm{VHS}>8+\mathrm{AX}+\mathrm{AU}$ & $10 / 21$ & 80 & 90.48 & 87.1 & 80 & 90.48 \\
\hline VHS $>8+A U+N T-p r o B N P$ & $10 / 17$ & 100 & 85 & 88.89 & 70 & 100 \\
\hline $\mathrm{VHS}>8.5+\mathrm{L} \times \mathrm{W}+\mathrm{AX}$ & $9 / 16$ & 75 & 73.68 & 74.07 & 54.55 & 87.5 \\
\hline VHS $>8.5+L \times W+N T-p r o B N P$ & $8 / 11$ & 100 & 84.62 & 89.47 & 75 & 100 \\
\hline $\mathrm{VHS}>8.5+\mathrm{L} \times \mathrm{W}+\mathrm{AU}$ & $7 / 21$ & 54.55 & 94.12 & 78.57 & 85.71 & 76.19 \\
\hline VHS $>8.5+\mathrm{L} \times \mathrm{W} / \mathrm{S} 4+\mathrm{AX}$ & $8 / 21$ & 71.43 & 82.61 & 80 & 55.56 & 90.48 \\
\hline VHS $>8.5+L \times W / S 4+N T-p r o B N P$ & $7 / 14$ & 100 & 88.24 & 90.91 & 71.43 & 100 \\
\hline $\mathrm{VHS}>8.5+\mathrm{L} \times \mathrm{W} / \mathrm{S} 4+\mathrm{AU}$ & $6 / 26$ & 50 & 95.45 & 81.25 & 83.33 & 80.77 \\
\hline VHS $>8.5+A X+N T-p r o B N P$ & $9 / 19$ & 100 & 90.48 & 92.86 & 77.78 & 100 \\
\hline $\mathrm{VHS}>8.5+\mathrm{AX}+\mathrm{AU}$ & $7 / 25$ & 75 & 95.83 & 90.63 & 85.71 & 92 \\
\hline VHS $>8.5+A U+N T-p r o B N P$ & $7 / 21$ & 100 & 95.45 & 96.43 & 85.71 & 100 \\
\hline $\mathrm{L} \times \mathrm{W}+\mathrm{AX}+\mathrm{NT}-$ proBNP & $14 / 10$ & 100 & 62.5 & 75 & 57.14 & 100 \\
\hline$L \times W+A X+A U$ & $12 / 15$ & 83.33 & 86.67 & 85.19 & 83.33 & 86.67 \\
\hline $\mathrm{L} \times \mathrm{W}+\mathrm{AU}+\mathrm{NT}-$ proBNP & $12 / 12$ & 100 & 68.75 & 79.17 & 61.54 & 100 \\
\hline $\mathrm{L} \times \mathrm{W} / \mathrm{S} 4+\mathrm{AX}+\mathrm{NT}-$ proBNP & $13 / 12$ & 100 & 66.67 & 76 & 53.85 & 100 \\
\hline$L \times W / S 4+A X+A U$ & $11 / 18$ & 81.82 & 88.89 & 86.21 & 81.82 & 88.89 \\
\hline $\mathrm{L} \times \mathrm{W} / \mathrm{S} 4+\mathrm{AU}+\mathrm{NT}-$ proBNP & $11 / 16$ & 100 & 84.21 & 88.46 & 70 & 100 \\
\hline$A X+A U+N T-p r o B N P$ & $10 / 17$ & 100 & 90 & 92.86 & 80 & 100 \\
\hline
\end{tabular}

$\mathrm{AX}=$ The subjective radiographic assessment of the left atrial size, $\mathrm{AU}=$ The ultrasonographic measurement of the left atrial diameter, $L \times W=$ The multiplication of the $L$ and the $W$ of the heart, $L \times W / S 4=$ The ratio of the multiplication of the $L$ and the $W$ of the heart to the $L$ of the fourth sternal thoracic bone, NPV $=$ Negative predictive value, NT-proBNP $=$ The NT-proBNP point-of-care test, $\mathrm{PPV}=$ Positive predictive value, VHS $>8=$ Vertebral heart score at a cutoff value of $>8$, VHS $>8.5=$ Vertebral heart score at a cutoff value of $>8.5$

Using the NT-proBNPpoint-of-care test in combination with the multiplication of the length and width of the heart assessed by radiography provided a higher percentage of accuracy. Using three tests together, including the NT-proBNP point-of-care test, multiplication of the length and width of the heart, and subjective radiographic assessment of the left atrial size or subjective ultrasonographic assessment of left 
Table-3: Specificity, sensitivity, accuracy, positive predictive, and negative predictive values of tests for distinguishing cats with $(n=22)$ and without congestive heart failure $(n=36)$.

\begin{tabular}{|c|c|c|c|c|c|c|}
\hline Parameters & $\begin{array}{c}\text { Number positive/ } \\
\text { negative }\end{array}$ & $\begin{array}{c}\text { Sensitivity } \\
(\%)\end{array}$ & $\begin{array}{l}\text { Specificity } \\
(\%)\end{array}$ & $\begin{array}{l}\text { Accuracy } \\
(\%)\end{array}$ & PPV & NPV \\
\hline \multicolumn{7}{|l|}{ One test } \\
\hline VHS $>8$ & $24 / 34$ & 63.63 & 72.22 & 68.97 & 58.33 & 76.47 \\
\hline VHS $>8.5$ & $13 / 45$ & 40.90 & 88.89 & 70.69 & 69.23 & 71.11 \\
\hline $\mathrm{L} \times \mathrm{W}$ & $24 / 34$ & 63.63 & 44.44 & 51.72 & 41.18 & 66.67 \\
\hline $\mathrm{L} \times \mathrm{W} / \mathrm{S} 4$ & $25 / 33$ & 50.00 & 61.11 & 56.89 & 44 & 66.67 \\
\hline AX & 29/29 & 72.72 & 63.89 & 67.24 & 55.17 & 79.31 \\
\hline NT-proBNP & $30 / 28$ & 81.81 & 66.67 & 72.41 & 60 & 85.71 \\
\hline$A U$ & $16 / 42$ & 59.10 & 91.67 & 79.31 & 81.25 & 78.57 \\
\hline \multicolumn{7}{|l|}{ Two tests } \\
\hline $\mathrm{VHS}>8+\mathrm{L} \times \mathrm{W}$ & $18 / 18$ & 68.75 & 65 & 66.67 & 61.11 & 72.22 \\
\hline $\mathrm{VHS}>8+\mathrm{L} \times \mathrm{W} / \mathrm{S} 4$ & $15 / 24$ & 58.82 & 77.27 & 69.23 & 66.67 & 70.83 \\
\hline $\mathrm{VHS}>8+\mathrm{AX}$ & $24 / 19$ & 75 & 74.07 & 74.42 & 63.16 & 83.33 \\
\hline VHS $>8+N T-p r o B N P$ & $15 / 19$ & 91.67 & 81.82 & 85.29 & 73.33 & 94.74 \\
\hline $\mathrm{VHS}>8+\mathrm{AU}$ & $11 / 29$ & 69.23 & 92.59 & 85 & 81.82 & 86.21 \\
\hline VHS $>8.5+L \times W$ & $11 / 22$ & 53.33 & 83.33 & 69.7 & 72.73 & 68.18 \\
\hline $\mathrm{VHS}>8.5+\mathrm{L} \times \mathrm{W} / \mathrm{S} 4$ & $9 / 28$ & 43.75 & 90.91 & 71.05 & 77.78 & 68.97 \\
\hline $\mathrm{VHS}>8.5+\mathrm{AX}$ & $13 / 29$ & 60 & 85.19 & 76.19 & 69.23 & 79.31 \\
\hline VHS > 8.5+NT-proBNP & $9 / 24$ & 77.78 & 91.67 & 87.88 & 77.78 & 91.67 \\
\hline $\mathrm{VHS}>8.5+\mathrm{AU}$ & $7 / 36$ & 50 & 96.77 & 83.72 & 85.71 & 83.33 \\
\hline$L \times W+A X$ & $21 / 15$ & 78.57 & 56.52 & 64.86 & 52.38 & 81.25 \\
\hline $\mathrm{L} \times \mathrm{W}+\mathrm{NT}-$ proBNP & $18 / 12$ & 81.25 & 64.29 & 73.33 & 72.22 & 75 \\
\hline$L \times W+A U$ & $15 / 23$ & 63.16 & 84.21 & 73.68 & 80 & 69.57 \\
\hline $\mathrm{L} \times \mathrm{W} / \mathrm{S} 4+\mathrm{AX}$ & $17 / 21$ & 66.67 & 69.57 & 68.42 & 58.82 & 76.19 \\
\hline L×W/S4+NT-proBNP & $14 / 17$ & 76.92 & 77.78 & 77.42 & 71.43 & 82.35 \\
\hline $\mathrm{L} \times \mathrm{W} / \mathrm{S} 4+\mathrm{AU}$ & $12 / 29$ & 56.25 & 88 & 75.61 & 75 & 75.86 \\
\hline$A X+N T-p r o B N P$ & $19 / 19$ & 86.67 & 73.91 & 78.95 & 68.42 & 89.47 \\
\hline$A X+A U$ & $12 / 25$ & 81.82 & 88.46 & 86.49 & 75 & 92 \\
\hline$A U+N T-p r o B N P$ & $13 / 25$ & 80 & 95.65 & 89.47 & 92.31 & 88 \\
\hline \multicolumn{7}{|l|}{ Three tests } \\
\hline $\mathrm{VHS}>8+\mathrm{L} \times \mathrm{W}+\mathrm{AX}$ & $15 / 13$ & 83.33 & 64.71 & 72.41 & 62.5 & 84.62 \\
\hline VHS $>8+L \times W+N T-p r o B N P$ & $12 / 9$ & 90.91 & 80 & 85.71 & 83.33 & 88.89 \\
\hline $\mathrm{VHS}>8+\mathrm{L} \times \mathrm{W}+\mathrm{AU}$ & $11 / 17$ & 69.23 & 86.67 & 78.57 & 81.82 & 76.47 \\
\hline $\mathrm{VHS}>8+\mathrm{L} \times \mathrm{W} / \mathrm{S} 4+\mathrm{AX}$ & $13 / 18$ & 69.23 & 77.78 & 74.19 & 69.23 & 77.78 \\
\hline VHS $>8+L \times W / S 4+N T-p r o B N P$ & $11 / 12$ & 90 & 84.62 & 86.96 & 81.82 & 91.67 \\
\hline $\mathrm{VHS}>8+\mathrm{L} \times \mathrm{W} / \mathrm{S} 4+\mathrm{AU}$ & $10 / 22$ & 66.67 & 89.47 & 80.65 & 80 & 80.95 \\
\hline VHS $>8+A X+N T-p r o B N P$ & $13 / 16$ & 90.91 & 83.33 & 86.21 & 76.92 & 93.75 \\
\hline $\mathrm{VHS}>8+\mathrm{AX}+\mathrm{AU}$ & $10 / 21$ & 88.89 & 90.91 & 90.32 & 80 & 95.24 \\
\hline VHS > 8+AU+NT-proBNP & $10 / 17$ & 100 & 94.44 & 96.3 & 90 & 100 \\
\hline $\mathrm{VHS}>8.5+\mathrm{L} \times \mathrm{W}+\mathrm{AX}$ & $9 / 16$ & 72.73 & 81.25 & 77.78 & 72.73 & 81.25 \\
\hline VHS $>8.5+L \times W+N T-p r o B N P$ & $8 / 11$ & 77.78 & 90 & 84.21 & 87.5 & 81.82 \\
\hline $\mathrm{VHS}>8.5+\mathrm{L} \times \mathrm{W}+\mathrm{AU}$ & $7 / 21$ & 50 & 93.75 & 75 & 85.71 & 71.43 \\
\hline $\mathrm{VHS}>8.5+\mathrm{L} \times \mathrm{W} / \mathrm{S} 4+\mathrm{AX}$ & $8 / 21$ & 58.33 & 88.89 & 76.67 & 77.78 & 76.19 \\
\hline VHS $>8.5+\mathrm{L} \times \mathrm{W} / \mathrm{S} 4+\mathrm{NT}-$ proBNP & $7 / 14$ & 75 & 92.86 & 86.36 & 85.71 & 86.67 \\
\hline $\mathrm{VHS}>8.5+\mathrm{L} \times \mathrm{W} / \mathrm{S} 4+\mathrm{AU}$ & $6 / 26$ & 45.45 & 95.24 & 78.13 & 83.33 & 76.92 \\
\hline VHS $>8.5+A X+N T-p r o B N P$ & $9 / 19$ & 77.78 & 89.47 & 85.71 & 77.78 & 89.47 \\
\hline$V H S>8.5+A X+A U$ & $7 / 25$ & 75 & 95.83 & 90.63 & 85.71 & 92 \\
\hline VHS $>8.5+A U+N T-p r o B N P$ & $7 / 21$ & 85.71 & 95.24 & 92.86 & 85.71 & 95.24 \\
\hline$L \times W+A X+N T-p r o B N P$ & $14 / 10$ & 83.33 & 66.67 & 75 & 71.43 & 80 \\
\hline$L \times W+A X+A U$ & $12 / 15$ & 81.82 & 81.25 & 81.48 & 75 & 86.67 \\
\hline $\mathrm{L} \times \mathrm{W}+\mathrm{AU}+\mathrm{NT}-$ proBNP & $12 / 12$ & 85.71 & 90 & 87.5 & 92.31 & 81.82 \\
\hline $\mathrm{L} \times \mathrm{W} / \mathrm{S} 4+\mathrm{AX}+\mathrm{NT}-$ proBNP & $13 / 12$ & 81.82 & 71.43 & 76 & 69.23 & 83.33 \\
\hline $\mathrm{L} \times \mathrm{W} / \mathrm{S} 4+\mathrm{AX}+\mathrm{AU}$ & $11 / 18$ & 80 & 84.21 & 82.76 & 72.73 & 88.89 \\
\hline $\mathrm{L} \times \mathrm{W} / \mathrm{S} 4+\mathrm{AU}+\mathrm{NT}-$ proBNP & $11 / 16$ & 81.82 & 93.33 & 88.46 & 90 & 87.5 \\
\hline$A X+A U+N T-p r o B N P$ & $10 / 17$ & 90 & 94.44 & 92.86 & 90 & 94.44 \\
\hline
\end{tabular}

$\mathrm{AX}=$ The subjective radiographic assessment of the left atrial size, $\mathrm{AU}=$ The ultrasonographic measurement of the left atrial diameter, $L \times W=$ The multiplication of the $L$ and the $W$ of the heart, $L \times W / S 4=$ The ratio of the multiplication of the $L$ and the $W$ of the heart to the $L$ of the fourth sternal thoracic bone, NPV $=$ Negative predictive value, NT-proBNP $=$ The NT-proBNP point-of-care test, $\mathrm{PPV}=$ Positive predictive value, VHS $>8=$ Vertebral heart score at a cutoff value of $>8$, VHS $>8.5=$ Vertebral heart score at a cutoff value of $>8.5$

atrial size, provided the best accuracy for diagnosing heart disease in cats.

The subjective ultrasonographic assessment of the left atrial size was the best method for diagnosing cats with HCM. A more accurate result was obtained when conducting the subjective ultrasonographic assessment of the left atrial size together with the NT-proBNP point-of-care test. The best tests for 
diagnosing HCM comprised the VHS at a cutoff value $>8.5$, subjective ultrasonographic assessment of the left atrial size, and NT-proBNP point-of-care test. This set of tests provided $96.43 \%$ accuracy.

The subjective ultrasonographic assessment of the left atrial size provided the highest percentage of accuracy. A higher percentage of accuracy was found when using a combination of tests, including the subjective ultrasonographic assessment of the left atrial size and NT-proBNP point-of-care test. Based on the results of this study, the best method to diagnose CHF in cats is to conduct a combination of tests, including VHS at a cutoff value $>8$, subjective ultrasonographic assessment of the left atrial size, and NT-proBNP point-of-care test.

\section{Discussion}

This study recommends the NT-proBNP point-of-care test or subjective ultrasonographic assessment of the left atrial size as the optimum methods for diagnosing cats with heart diseases, HCM, or CHF, particularly when comprehensive echocardiography is unavailable. The previous studies suggested that the NT-proBNP point-of-care test can be used to assess the severity of heart disease and HCM in cats $[14,15]$. Moreover, the NT-proBNP point-of-care test may be used as a complementary test to evaluate the risk of death [16] and distinguish respiratory distress with cardiogenic and non-cardiogenic causes in cats $[9,17]$.

The usefulness of NT-proBNP in the diagnosis of heart disease in cats has been reviewed [18]. The use of the NT-proBNP point-of-care test has some limitations: The blood sampling protocol requires centrifugation, separation, and storage of plasma within a limited time after sample collection [15]. A previous study showed that a negative result of the NT-proBNP point-of-care test could not exclude the presence of underlying heart disease [19]. In contrast, performing the subjective ultrasonographic assessment of the left atrial size is less stressful for cats than using the NT-proBNP point-of-care test because a lower degree of restraint is required [20].

The assessment of heart size on radiographs may not be suitable as a sole method for diagnosing heart diseases in cats. All radiographic assessment methods used in this study, including VHS, multiplication of the length and width of the heart, and subjective radiographic assessment of the left atrial size provided moderate sensitivity and specificity in diagnosing heart diseases, HCM, and CHF in cats. In some regions, animal hospitals generally perform thoracic radiography to diagnose heart disease in cats. However, some conditions may make interpretation of the radiographs more challenging $[5,21]$, such as concurrent respiratory disease, heartworm disease, presence of non-cardiogenic pleural effusion, radiographic positioning, pericardial fat, and the phase of the respiratory and cardiac cycles [22]. In addition, the left atrial enlargement assessed by thoracic radiography may be absent in acute $\mathrm{CHF}$ in some cats [23]. For a more accurate diagnosis of heart disease in cats, we recommend using radiography in combination with other methods, such as NT-proBNP point-of-care test and subjective ultrasonographic assessment of the left atrial size.

Other tests that can help in the diagnosis of HCM in cats have been proposed, including genetic tests, other biomarkers such as cardiac troponin I, and cardiac arrhythmia test [24]. The mutation of myosin-binding protein C (MYBPC3) has been found in Maine Coon and Ragdoll cats affected with HCM $[25,26]$. However, not all cats with MYBP3 mutation have HCM. Moreover, some cats with MYBPC3 mutation may not develop HCM [27]. Cardiac troponin I is one of the cardiac biomarkers that may be used in test for the diagnosis of HCM with high sensitivity (91.7\%) and specificity (95.4\%) [28]. Plasma growth differentiation factors 8 and 11, biomarkers associated with cardiac hypertrophy in humans, have been studied in cats. However, both growth differentiation factors 8 and 11 cannot be used to differentiate normal cats and cats with HCM [29]. A previous study showed that ventricular arrhythmias are common in cats with HCM, suggesting that arrhythmia may be used as a complementary method for diagnosing heart disease in cats [30].

The limitations of the study were the small number of cats included. In addition, the use of radiographic measurement results and interpretation from five individuals rather than one may have affected the accuracy of radiography.

\section{Conclusion}

The results of this study show that the more tests used, the more reliable the diagnosis of cats with heart disease, HCM, and CHF. However, using a combination of methods has some limitations, including the availability and cost of each test. Selecting which tests to perform depends on the purpose of diagnosis. All methods used in this study are intended for use as a first step diagnosis; therefore, echocardiography is still needed to confirm the type of heart disease in cats.

\section{Authors' Contributions}

TL, NR, KS, PS and TT : data collection, data analysis, and writing the first draft; NR: SDS: supervision, data validation, writing review, and editing. All authors read and approved the final manuscript.

\section{Acknowledgments}

This study was supported in part by the grant from the academic affair, Faculty of Veterinary Science, and Chulalongkorn University. The authors would like to thank the Small Animal Hospital, Faculty of Veterinary Science, and Chulalongkorn University for supporting data and facilities. Special thanks to Dr. Vachira Hunprasit and Prof. Dr. Padet Tummarak for statistical assistance.

\section{Competing Interests}

The authors declare that they have no competing interests. 


\section{Publisher's Note}

Veterinary World remains neutral with regard to jurisdictional claims in published institutional affiliation.

\section{References}

1. Buchanan, J.W. (1999) Prevalence of cardiovascular disorders. In: Fox, P.R., Sisson, D.D. and Moise, N.S., editor. Textbook of Canine and Feline Cardiology: Principles and Clinical Practice. $2^{\text {nd }}$ ed. WB Saunders, Philadelphia, PA. p457-470.

2. Payne, J.R., Brodbelt, D.C. and Fuentes, V.L. (2015) Cardiomyopathy prevalence in 780 apparently healthy cats in rehoming centers (the CatScan study). J. Vet. Cardiol., 17 Suppl 1: 244-257.

3. Wess, G., Daisenberger, P., Mahling, M., Hirschberger, J. and Hartmann, K. (2011) Utility of measuring plasma $\mathrm{N}$-terminal pro-brain natriuretic peptide in detecting hypertrophic cardiomyopathy and differentiating grades of severity in cats. Vet. Clin. Pathol., 40(2): 237-244.

4. Silverstein, D.C. and Hopper, K. (2015) Feline cardiomyopathy. In: Silverstein, D.C. and Hopper, K., editor. Small Animal Critical Care Medicine. $2^{\text {nd }}$ ed. Elsevier, St. Louis. p218-225.

5. Sleeper, M.M., Roland, R. and Drobatz, K.J. (2013) Use of the vertebral heart scale for differentiation of cardiac and noncardiac causes of respiratory distress in cats: 67 cases (2002-2003). J. Am. Vet. Med. Assoc., 242(3): 366-371.

6. Fucharoen, P., Pankham, W., Thong-In, S., Juengthanasomboon, S. and Surachetpong, S.D. (2017) New methods to differentiate cardiac and noncardiac disease cats from thoracic radiographs. Thai J. Vet. Med., 47(2): 199-205.

7. Payne, J.R., Borgeat, K., Connolly, D.J., Boswood, A., Dennis, S., Wagner, T., Menaut, P., Maerz, I., Evans, D., Simons, V.E., Brodbelt, D.C. and Fuentes, V.L. (2013) Prognostic indicators in cats with hypertrophic cardiomyopathy. J. Vet. Intern. Med., 27(6): 1427-1436.

8. Weber, M., Mitrovic, V. and Hamm, C. (2006) B-type natriuretic peptide and N-terminal pro-B-type natriuretic peptide diagnostic role in stable coronary artery disease. Exp. Clin. Cardiol., 11(2): 99-101.

9. Connolly, D.J., Magalhaes, R.J.S., Syme, H.M., Boswood, A., Fuentes, V.L., Chu, L. and Metcalf, M. (2008) Circulating natriuretic peptides in cats with heart disease. $J$. Vet. Intern. Med., 22(1): 96-105.

10. Sangster, J.K., Panciera, D.L., Abbott, J.A., Zimmerman, K.C. and Lantis, A.C. (2014) Cardiac biomarkers in hyperthyroid cats. J. Vet. Intern. Med., 28(2): 465-472.

11. Kellihan, H.B., Mackie, B.A. and Stepien, R.L. (2011) NT-proBNP, NT-proANP and cTnI concentrations in dogs with pre-capillary pulmonary hypertension. J. Vet. Cardiol., 13(3): 171-182.

12. Fox, P., Basso, C., Thiene, G. and Maron, B.J. (2014) Spontaneously occurring restrictive nonhypertrophied cardiomyopathy in domestic cats: A new animal model of human disease. Cardiovasc. Pathol., 23(1): 28-34.

13. Scansen, B.A., Schneider, M. and Bonagura, J.D. (2015) Sequential segmental classification of feline congenital heart disease. J. Vet. Cardiol., 17 Suppl 1: S1-S52.

14. Machen, M.C., Oyama, M.A., Gordon, S.G., Ruch, J.E., Achen, S.E., Stepien, R.L., Fox, P.R., Saunders, A.B., Cunningham, S.M., Lee, P.M. and Kellihan, H.B. (2014) Multi-centered investigation of a point-of-care NT-proBNP ELISA assay to detect moderate to severe occult (pre-clinical) feline heart disease in cats referred for cardiac evaluation. J. Vet. Cardiol., 16(4): 245-255.

15. Hsu, A., Kittleson, M.D. and Paling, A. (2009) Investigation into the use of plasma NT-proBNP concentration to screen for feline hypertrophic cardiomyopathy. J. Vet. Cardiol., 11
(Suppl 1): 63-70.

16. Maisel, A.S., Bhalla, V. and Braunwald, E. (2006) Cardiac biomarkers: A contemporary status report. Nat. Clin. Pract. Cardiovasc. Med., 3(1): 24-34.

17. Fox, P.R., Oyama, M.A., Reynolds, C., Ruch, J.E., DeFrancesco, T.C., Keene, B.W., Atkins, C.E., Macdonald, K.A., Schober, K.E., Bonagura, J.D., Stepien, R.L., Kellihan, H.B., Nguyenba, T.P., Lehmkuhl, L.B., Lefborn, B.K., Moise, N.S. and Hogan, D.F. (2009) Utility of plasma N-terminal pro-brain natriuretic peptide (NT-proBNP) to distinguish between congestive heart failure and noncardiac causes of acute dyspnea in cats. J. Vet. Cardiol., 11 Suppl 1: 51-61.

18. De Lima, G.V., Ferreira, F.D.S. (2017) N-terminal-pro brain natriuretic peptides in dogs and cats: A technical and clinical review. Vet. World., 10(9): 1072-1082.

19. Harris, A.N., Beatty, S.S., Estrada, B., Winter, B., Bohannon, M., Sosa, I., Hanscorn, J., Mainville, C.A. and Gallagher, A.E. (2017) Investigation of an N-terminal prohormone of brain natriuretic peptide point-of-care ELISA in clinically normal cats and cats with cardiac disease. $J$. Vet. Intern. Med., 31(4): 994-999.

20. Lisciandro, G.R. (2014) The thoracic FAST3 (TFAST3) exam. In: Lisciandro, G.R., editor. Focused ultrasound techniques for the small animal practitioner. $1^{\text {st }}$ ed. Wiley Blackwell, Ames. p140-165.

21. Benigni, L., Morgan, N. and Lamb, C.R. (2009) Radiographic appearance of cardiogenic pulmonary edema in 23 cats. J. Small Anim. Pract., 50(1): 9-14.

22. Guglielmini, C., Toaldo, M.B., Poser, H., Menciotti, G., Cipone, M., Cordella, A. Contiero, B. and Diana, A. (2014) Diagnostic accuracy of the vertebral heart score and other radiographic indices in the detection of cardiac enlargement in cats with different cardiac disorders. J. Feline Med. Surg., 16(10): 812-825.

23. Schober, K.E., Welti, E. and Drost, W.T. (2014) Radiographic and echocardiographic assessment of left atrial size in 100 cats with acute left-sided congestive heart failure. Vet. Radiol. Ultrasound., 55(4): 359-367.

24. Häggström, J., Fuentes, V.L. and Wess, G. (2015) Screening for hypertrophic cardiomyopathy in cats. J. Vet. Cardiol., 17 Suppl 1: S134-S149.

25. Meurs, K.M., Sanchex, X., David, R.M., Bowles, N.E., Towbin, J.A., Reiser, P.J., Kittleson, J.A., Munro, M.J., Dryburgh, K., Macdonald, K.A. and Kittleson, M.D. (2005) A cardiac myosin binding protein $\mathrm{C}$ mutation in the Maine coon cat with familial hypertrophic cardiomyopathy. Hum. Mol. Genet., 14(23): 3587-3593.

26. Meurs, K.M., Norgard, M.M., Ederer, M.M., Hendrix, KP. and Kittleson, M.D. (2007) A substitution mutation in the myosin-binding protein $\mathrm{C}$ gene in Ragdoll hypertrophic cardiomyopathy. Genomics, 90(2): 261-264.

27. Wess, G., Schinner, C., Weber, K., Kuchenhoff, H. and Hartmann, K. (2010) Association of A31P and A74T polymorphisms in the myosin binding protein $\mathrm{C} 3$ gene and hypertrophic cardiomyopathy in Main coon and other breed cats. J. Vet. Intern. Med., 24(3): 527-532.

28. Hertzsch, S., Roos, A. and Wess, G. (2019) Evaluation of a sensitive cardiac troponin I assay as a screening test for the diagnosis of hypertrophic cardiomyopathy in cats. J. Vet. Intern. Med., 33(3): 1242-1250.

29. Yang, V.K., Rush, J.E., Bhasin, S., Wagers, A.J. and Lee, R.T. (2019) Plasma growth differentiation factors 8 and 11 levels in cats with congestive heart failure secondary to hypertrophic cardiomyopathy. J. Vet. Cardiol., 25: 41-51.

30. Bartoszuk, U., Keene, BW., Baron Toaldo, M., Pereira, N., Summerfield, N., Novo Matos, J. and Glaus, T.M. (2019) Holter monitoring demonstrates that ventricular arrhythmias are common in cats with decompensated and compensated hypertrophic cardiomyopathy. Vet. J., 243: 21-25. 